\title{
Multi-Agent Geosimulation In Support To "What if” Courses Of Action Analysis
}

\author{
Hedi Haddad \\ Laval University \\ Computer Science Department, 3904 \\ Pav. Pouliot, Québec, G1K 7P4 \\ 1-888-569-7979 \\ hedi.haddad.1@ulaval.ca
}

\author{
Bernard Moulin \\ Laval University \\ Computer Science Department, 3904 \\ Pav. Pouliot, Québec, G1K 7P4 \\ 1-888-569-7979 \\ bernard.moulin@ift.ulaval.ca
}

\begin{abstract}
In this paper we propose an approach to support "what-if" analysis in the context of COA evaluation. Our approach consists in using multiagent geosimulation to simulate the execution of COAs in a Virtual Geographic Environment (VGE) which can change during the simulation, and then allowing the user to explore various assumptions and to analyse their outcomes. We identify the requirements to support this approach and we present how we implement them in the MAGS-COA software. We also illustrate our approach on an example and we present future works.
\end{abstract}

\section{Categories and Subject Descriptors}

I.6.6 [Simulation and Modeling]: Simulation Output Analysis; I.2.11 [Artificial Intelligence]: Distributed Artificial Intelligence Multiagent systems; I.2.4 [Artificial Intelligence]: Knowledge Representation Formalisms and Methods - Semantic networks.

\section{General Terms}

Algorithms, Design, Experimentation, Theory.

\section{Keywords}

Multiagent geosimulation, "What if" analysis, COA evaluation.

\section{INTRODUCTION}

We are interested in the evaluation of courses of action (COAs) to be executed in a dynamic geographic environment. A course of action is an outline of a plan specifying the manner in which a set of resources might attempt to accomplish a mission [9]. In the context of geographic environments, the characteristics of the geographic space and the different dynamic phenomena that may occur in it must be taken into account during the evaluation of COAs. However, detailed information about the geographic space is usually not available, and the dynamic phenomena are unpredictable. Thus, geographic reasoning is typically uncertain and based on incomplete information. In these conditions, it has been proven that a "What-if" analysis is the most suitable and the

Permission to make digital or hard copies of all or part of this work for personal or classroom use is granted without fee provided that copies are not made or distributed for profit or commercial advantage and that copies bear this notice and the full citation on the first page. To copy otherwise, or republish, to post on servers or to redistribute to lists, requires prior specific permission and/or a fee.

SIMUTOOLS 2008, March 03-07, Marseille, France

Copyright $\odot 2008$ ICST 978-963-9799-20-2

DOI 10.4108/ICST.SIMUTOOLS2008.3026 most used reasoning strategy by experts, especially when it is either impossible or impractical to conduct a physical experiment [43]. "What if" reasoning is a kind of qualitative reasoning based on a mental model and allowing to reason with partial knowledge and thus to deal with the ambiguity inherent in situations of uncertainty $[17,43]$. Considering the evaluation of COAs, "What if" reasoning allows the planner to think about the implications of different assumptions by playing out different scenarios and then by evaluating the plausibility of their consequences [43]. According to [10], "what if' thinking is a three-step mental simulation that consists of 1) visualizing some initial situation, 2) carrying out one or more operations (assumptions) on it and 3), seeing what happens. During the third step, causal reasoning occurs to explain the results of the manipulations of the second step.

Our review of the literature showed that there is a lack of decision support systems that can support all the steps of COAs "what if" analysis in the context of dynamic geographic environments. In this paper we propose a multi-agent geosimulation-based approach to support a such analysis. In Section 2, we present the general principles of our approach and in Section 3 we discuss the requirements in order to develop it. In Section 4 we present the MAGS-COA tool, developed as a proof of concept of the proposed approach. In Section 5 we apply our approach to the domain of critiquing systems and we illustrate it by an example. In Section 6 we discuss our contributions in relation to similar works, present future work and conclude.

\section{A GENERAL APPROACH TO SUPPORT COAs "WHAT-IF" ANALYSIS IN GEOGRAPHIC ENVIRONMENTS}

Our goal is to propose and develop an approach to support a "what-if" analysis of COAs to be executed in a dynamic geographic environment. Supporting "what-if" thinking requires to support its three steps, i.e. allowing a person 1) to specify and visualize some initial situation, 2) to specify some operations corresponding to different assumptions made about this situation and 3) to execute and analyse the consequences of these operations on this situation. In addition, supporting the evaluation of COAs in the context of dynamic geographic environments requires the ability to represent the resources involved in the COA, to specify and execute how they must operate in order to achieve their mission, to model the geographic environment, to represent and execute the different dynamic phenomena that may occur in it, and to model and explain how the resources of the $\mathrm{COA}$ are influenced by this dynamic environment. 
In order to meet these requirements, we propose to use a multiagent geosimulation-based approach consisting in simulating the execution of COAs in a Virtual Geographic Environment $(V G E)$ which can change during the simulation, and then allowing the user to explore various assumptions and to analyse their outcomes. Geosimulation is a relatively recent domain that is characterized by an explicit attention to space and geography [41] while multiagent geosimulation consists in simulating agent's behaviors in VGEs [30, 34], using data from geographic information systems (GIS). In recent years, multiagent geosimulation has been used to model several social phenomena, especially mobility in urban environments, such as in $[2,5,6,19$, $26,42,45]$, to mention a few. We think that multiagent geosimulation- by integrating technological advances of autonomous agents, GIS data and dynamic phenomena modelingis appropriate in the context of the "What if" analysis of COAs. First, it can be a good support to the "what if" mental simulation. Second, it provides a somewhat analog representation of the geographic reality and its dynamism. Finally, it is a good way to represent the dynamism corresponding to the behaviours of the resources involved in the COA and their interactions. Hence, our approach is composed of three steps (Figure 1). In the first step, the user specifies his COA (the different resources, their initial locations in the VGE and their tasks to achieve the mission) as well as the different operations corresponding to his assumptions. The second step consists in using a multiagent-geosimulation system to simulate the COA in a $V G E$. The resources of the COA are represented by software agents that are inserted in the $V G E$ and autonomously carry out their activities. They react to the actions of other agents, they are constrained by the characteristics of the $V G E$ and they are influenced by the effects of several "happenings" or events that occur in it (as for example, a flood caused by heavy rainfall). The unfolding of the geosimulation is captured and saved in data structures in order to be analysed in the third step.

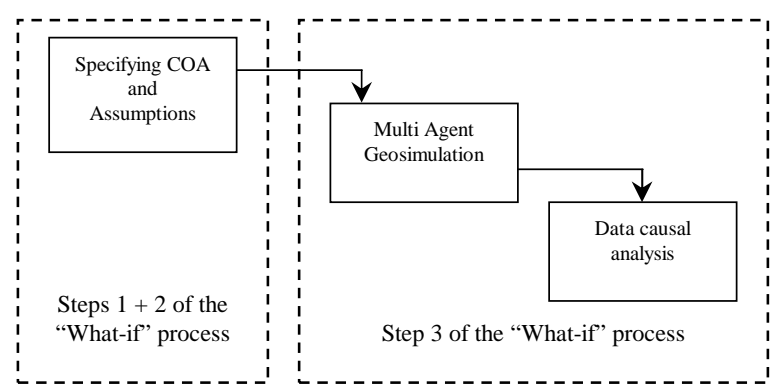

Figure 1. A general approach to support COAs "Whatif" analysis

The third step consists in analysing the data generated by the multiagent geosimulation step. As we aim to support "what if" analysis, we are particularly interested in causal reasoning and in identifying the causal relationships between the user's assumptions and geosimulation results. This kind of analysis requires a qualitative reasoning strategy, and thus classical analysis techniques (such as statistical and mathematical techniques) of simulations are not appropriate. There is a need for qualitative analysis approaches. In the case of causal reasoning, we must first semantically define and explicitly represent the elements describing the unfolding of the geosimulation. Second, we must be able to collect information about these elements during the geosimulation. Finally, the system must have the necessary ontological knowledge allowing to identify causal relationships between these elements.

In the next section we present the requirements needed to develop our approach and we detail each of its steps.

\section{REQUIREMENTS FOR USING MULTIAGENT GEOSIMULATION IN SUPPORT TO QUALITATIVE COA ANALYSIS}

Supporting the approach that we propose requires a multiagent geosimulation environment that enables the user 1) to specify COAs and assumptions, 2) to simulate them in a $V G E$ and 3) to analyse causal relationships between the assumptions and the simulation results. In the following we present these requirements.

\subsection{Scenario Specification}

We call a scenario the description of both a COA and the set of related assumptions specified by the user. The description of a COA indicates the initial positions of the involved resources in the $V G E$ and shows how (which tasks or goals need to be carried out), when (temporal constraints) and where (spatial positions) they must achieve a given mission. Assumptions correspond to the different "happenings" that may occur in the VGE and that are not caused by the resources' intentional actions, as for example rain falls and movements of fog patches. The scenario specification must rely on two kinds of ontological knowledge. On one hand, there is specific-domain knowledge describing the resources participating in the COA and the tasks that they are able to carry out. On the other hand, there is knowledge describing the $V G E$ and its natural physical phenomena. Examples include different kinds of flows (heat, liquid, gas, etc.), phase changing (boiling, freezing) as well as natural happenings (rain, fog, etc.) and their effects. We present this knowledge in the following subsection.

\subsection{Multiagent Geosimulation}

Applying multiagent geosimulation to support the qualitative analysis of COAs implies three main requirements. First, we need to represent the $V G E$ and to simulate its natural dynamic phenomena. Second, we need autonomous agents that are located in this $V G E$ and that can perceive it, react to its changes and operate in it in order to achieve a given mission. Third, we need to collect data about the evolution of the simulation that is required to carry out a qualitative causal analysis.

\subsubsection{A Dynamic Virtual Geographic Environment}

The structure of geographic environments has been widely studied by the geographic ontologies research community. Geographic ontologies aim to "produce an account of the entities existing in the world, of the types or categories under which these entities fall, and of the different sorts of relations which hold between them" [21]. According to Grenon and Smith [21], we may distinguish two modes of existence for entities populating the world. The first mode corresponds to an 'endurant' view according to which there are entities "that have continuous existence and a capacity to endure through time even while undergoing different sorts of changes". The second mode 
corresponds to an occurrent view that describes occurrent entities that "occur in time and unfold themselves through a period of time". Similarly to this classification, we distinguish a static and a dynamic views of the $V G E$.

\subsubsection{Static View}

We push further the works of the geographic ontologies research community in order to organise our static VGE using the concepts of Space, spatial zones, geographic objects and geographic portions (Figure 2).

\section{Space and Spatial zones}

We adopt the definition of Space and spatial zone proposed in [21]. In our model Space is the entire spatial universe (the maximal spatial region) and all spatial zones are parts of it. However, we use a different partition of Space. At a first elementary level, the Space is partitioned into a set of regular cells called pixels. Then, spatial zones are incrementally constructed in Space. A spatial zone is associated with a set of pixels. At a second level, Space is completely partitioned into a set of adjacent spatial zones in a manner that Space is totally covered. Let $n$ be the number of spatial zones of Space, we have: Space $=\bigcup_{i}^{n} Z_{i}$. Spatial zones are used as a reference framework to localize geographic objects in Space.

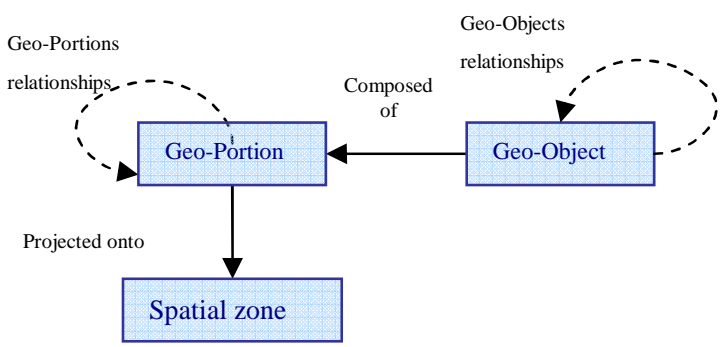

Figure 2. Structure of the static $V G E$

\section{Geo-Object and Geo-Portion}

Based on cognitive studies, the geographic ontologies research community organizes the static geographic environment in a set of geographic objects. Geographic objects are "spatial objects on or near the surface of the earth. They are objects of a certain minimal scale, they are typically complex, and they have parts and boundaries" [33]. Geographic objects can be "regions, parcels of land and water-bodies, topographic features such as bays, promontories, mountains and canyons, hills and valleys, roads, buildings, bridges, as well as the parts and aggregates of all of these". Because geographic objects are non movable, they are located in Space [15]. We use the term Geo-Object to designate a geographic object [15]. In our model, a Geo-Object may be composed of several parts and not only of an interior and a border as in [15]. We introduce the new concept of Geo-Portion to represent these portions. A Geo-Portion has a type and belongs to only one Geo-Object. A Geo-Object may be composed of one or several Geo-Portions. For example, a river may be represented as a Geo-Object composed of several Geo-Portions. This decomposition is necessary if we want to qualitatively simulate the propagation of information in the space, such as a pollution area travelling downstream in river. A Geo-Portion is projected onto only one spatial zone in Space. Spatial zones are used to locate Geo-Portions in Space. The form and the size of a spatial zone depend on the form and the size of its equivalent GeoPortion which, in turn, depends on the used spatial model (for example, vector or raster model in a GIS).

\section{Geo-Object / Geo-Object and Geo-Portion / Geo-Portion relationships}

The $V G E$ is enriched with ontological knowledge describing - in addition to the semantics and the characteristics of the geoportions- the different relationships between Geo-Objects and Geo-Portions, such as topological, superposition and proximity relationships. See [22, 23] for more details.

\subsubsection{Dynamic View}

In addition to represent the static elements of a $V G E$, we need to simulate different dynamic phenomena that may occur in it. Dynamic phenomena are modeled using the concept of change. However, changes in the $V G E$ could be caused by agents (resources of the COA) or by natural "happenings". Hence, simulating dynamic phenomena consist in simulating these two kinds of change. Changes caused by agents are embedded in the models used to develop the behaviors of these agents. We present these models in the next subsection. Changes caused by natural "happenings" (such as the characteristics of fog in reducing visibility and the force of wind in changing the direction of sea waves) can be simulated using either quantitative or qualitative models. Quantitative models are usually based on mathematical models that capture some aspects of real phenomena. The limit of these models is that they require precise and realistic data about the simulated phenomena. This data is often difficult to collect (such as collecting data about volcanoes' emissions), and the models are difficult to validate. By contrast, Qualitative Simulation (QS) is used to predict a set of possible behaviors of the modeled phenomena based on a qualitative model of the world [31]. The power of QS comes from "its ability to express natural types of incomplete knowledge of the world, and the ability to derive a provably complete set of possible behaviors in spite of the incompleteness of the model". While quantitative approaches are interesting, in our project we decided to begin with qualitative models to simulate natural phenomena in virtual geographic environments for two reasons. First, as we mentioned in the introduction, geographic reasoning is based on incomplete information. Second, it has been demonstrated that human beings reason qualitatively about the geographic space [12]. Later, depending of the application domain and the available data, quantitative models may be used to simulate some natural phenomena, such as mathematical models of flood [25] and soil erosion [37].

We illustrate a simple qualitative simulation approach using the example of turbulence zones as treated by aviation experts [3]. In fact, in the domain of aviation, turbulence in mountainous areas may be a source of danger for certain flying resources (such as helicopters and small aircrafts). The position and the intensity of turbulence areas depend of the wind's speed and orientation, the steepness of the mountain slope and the form of the ridge. For example, Figure 3 illustrates the position of the turbulence area created by a moderate west-east wind ( 11 to 20 knots) on a snake ridge, while Figure 4 illustrates the position of such an area created on a crown ridge [3]. 


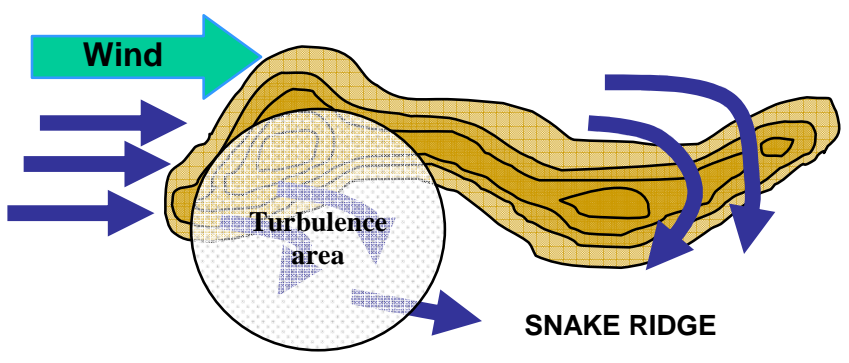

Figure 3. Turbulence areas in Snake ridge [3]

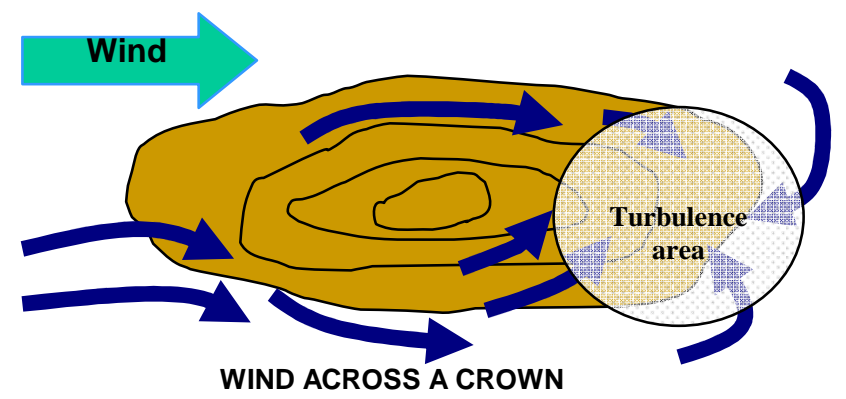

Figure 4. Turbulence areas in Crown ridge [3]

In this simple example, we focus on the turbulence areas created by the wind. Qualitatively simulating the effect of the happening of type wind requires to specify the wind's orientation and speed so that, depending on the ridge shape, qualitative turbulence areas will be created in the $V G E$. These areas are represented by reactive objects. Reactive objects are reactive agents that we use to qualitatively simulate the behaviors of natural physical phenomena. For example, the turbulence area is simulated by a reactive object characterized by an intensity and spatial stretch, and having as a behavior to apply on aircrafts strong updrafts and downdrafts (for example). Using this simple mechanism, we can qualitatively simulate the effects of the wind on both the $V G E$ and the resources involved in the COA. In Section 5 we present another example in which the wind is also simulated by a reactive object.

\subsubsection{Autonomous agents}

Executing a COA in a $V G E$ requires to use agents that are able to perceive the $V G E$ and to autonomously react to its changes. These requirements are met by using the MAGS platform [34] as a multi-agent geosimulation environment. MAGS agents are equipped with perception, navigation and behavioral capabilities.

With respect to the perception capabilities, an agent has a perception field which enables it to perceive 1) terrain features such as elevation and slopes, 2) the geographic objects and the other agents located in the agent's range of perception, and 3) dynamic areas or volumes whose shapes change during the simulation (such us smoky or foggy areas) [34].

Concerning the navigation capabilities, MAGS agents may use two navigation modes: Following-a-path-mode in which agents follow specific paths such as roads or Obstacle-avoidance-mode in which the agents move through open spaces avoiding obstacles.

Finally, in the MAGS platform, an agent is associated with a set of objectives that it tries to reach. The objectives are organized in hierarchies composed of nodes representing composite objectives and leaves representing elementary objectives associated with actions that the agent can perform. Further details about agents capabilities in the MAGS platform can be found in [34].

\subsubsection{Data collection and observer agents}

In order to analyze the causal relationships between the assumptions specified in the scenario and the results of the geosimulation, we need to collect data about the evolution of the simulation in the $V G E$. For this purpose, we use a special kind of agents called observer agents. Currently, our observer agents collect information about the following types of information:

a) The change of value of a Concurrent Condition:

We use Concurrent Conditions to represent constraints that must hold during the execution of an agent's objective, while preconditions must only hold before the objective's activation. When a constraint on a concurrent condition is violated, the objective automatically fails. For example, the plane's flying objective fails whenever the level of fuel reaches zero.

b) The change of a status of an agent's objective:

This information gives the exact time at which the status of an agent's objective changes value. The status of an objective can take the following values: 1) Goal-Start when the objective begins to be executed, 2) Completed-With-Success when the objective is completely executed with success, 3) Completed-With-Failure if the objective is completely executed without reaching its expected effect and 4) Interrupted if the execution of the objective is temporarily stopped.

c) Exit-from and entry-into spatial areas:

This information describes at which instant a given agent enters, exits or gets closer (within a given distance) to a given GeoObject, Geo-Portion or Reactive Object (such as agents simulating turbulence and foggy areas).

d) A change of a state value:

This information describes at which instant a state of an entity of the world (agents, geo-portions, geo-objects or smart objects) changes value.

\subsection{Data analysis and causal reasoning}

As we mentioned in Section 2, classical techniques usually used to analyze simulations results are not appropriate in the context of qualitative causal analysis. In order to support such an analysis, we think that there is a need for new techniques which must fulfill three requirements. First, we need to explicitly model and represent the elements describing the evolution of the simulation. Second we need to collect data about these elements during the simulation. Finally, we need appropriate knowledge to infer causal relationships between these elements. In the last section we presented how we use observer agents to fulfill the second requirement, i.e. collecting data about the evolution of the simulation. In this section we present how we addressed the first and third requirements.

\subsubsection{Modeling the evolution of the simulation}

In Section 3.2.1.2 we showed that dynamic phenomena are modeled using the concept of change. Thus, the evolution of the simulation can be described as a succession of changes ordered 
according to the time axis. In fact, as elaborated by [38], we agree that "the passage of time is important only because changes are possible with time" and that "the concept of time would become meaningless in a world where no changes were possible". Changes used to model dynamic phenomena have been widely studied by different research communities (such as temporal logics and GIS communities), and several solutions were proposed to model them. In [22] we proposed a new model to represent dynamic phenomena in geographic environments. In this model, a dynamic phenomenon is described using cognitive archetypes [13] which are structures describing a change as a transition of the world from an initial situation $\mathrm{Sit}_{1}$ to another posterior situation $\mathrm{Sit}_{2}$. The transition comprises three temporal zones: before transition $\left(\mathrm{Sit}_{1}\right)$, during transition from $\mathrm{Sit}_{1}$ to $\mathrm{Sit}_{2}$, and after transition ( Sit $_{2}$ ). Similarly to Desclés' approach [13], in our model a dynamic phenomenon can be either an event (transition with negligible duration) or a process (transition with significant duration). However, the event is the elementary change: a process is marked by two events indicating respectively its beginning and its end. We formalize dynamic phenomena using conceptual graphs [40], a knowledge representation formalism known to express meaning in a form that is logically precise and computationally tractable. Syntactically, a conceptual graph (C.G) is a network of concept nodes linked by relation nodes. Concept nodes are represented by the notation [Concept Type: Concept instance] and relation nodes by (RelationshipName). The concept instance can either be a value, a set of values or a CG. A CG can be represented in either graphical or linear notations. In the graphical notation, concepts are represented by rectangles, relations by circles and the links between concept and relation nodes by arrows. The linear notation (or linear form) is more compact than the graphical one and uses square brackets instead of boxes and parentheses instead of circles. Further details about how we use cognitive archetypes and CGs to model dynamic phenomena can be found in $[22,23]$. We use this formalism to represent data collected by the observer agents during the simulation. For example, Figure 5 illustrates the representation of the event describing the entry of an agent into a spatial zone. The geographic environment corresponds to an urban environment. In Section 5 we present another example of a large-scale geographic environment.

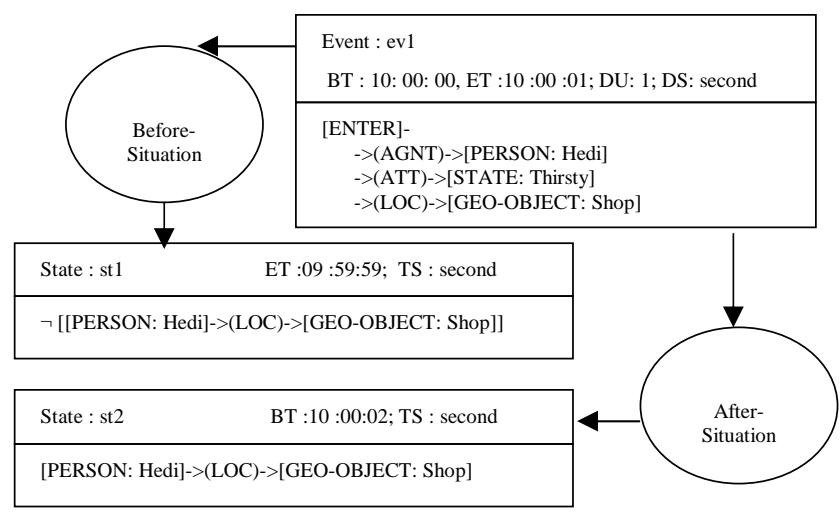

Figure 5. An example of event representation

The event is identified by $e v l$, for reference purposes. It describes the fact that the person Hedi is thirsty and enters the shop.
Temporal information is associated with the event showing its beginning time (BT: 10:00:00), ending time (ET: 10:00:01), duration (DU: 1) and duration scale (DS: Second). The event triggers a change from a "before event situation" to an "after event situation". The first situation describes a static state identified by st1. It has only two time parameters: ending time (ET: 09:59:59) and time scale (TS: second). It describes the fact that Hedi is somewhere out side the shop. This state is related to the event $e v 1$ by the Before-Situation relationship. The second situation is a static state identified by st2. It also has only two time parameters: beginning time (BT: 10:00:02) and time scale (TS: second). It describes the fact that Hedi is located in the shop. This state is related to the event $e v l$ by the After-Situation relationship.

\subsubsection{Causal analysis of dynamic phenomena}

The result of the multiagent geosimulation is a set of independent events representing the changes reported by the observer agents (observations). There is not any semantic relationship between these instances yet. Our objective is to establish a causal relationship between them. Let us denote by $\operatorname{Cause}\left(e_{e}\right)=e_{c}$ this causal relationship, where $e_{c}$ is the cause event and $e_{e}$ the effect event. Proposing formal models to infer causality is known to be a difficult task, and although causal reasoning has been studied by the AI research community for several years, it is still an active research problem [46]. Especially, the study of causal relationships between dynamic phenomena in a spatial environment is a relatively recent research trend [7, 47]. We push further works of the causal reasoning research community to define two kinds of constraints that must hold in order to meet a causal relationship: spatio-temporal and semantic constraints.

Spatio-temporal constraints are derived from the fact that human recognition of causal relations is based on recognition of precedence and contiguity between the cause and the effect [29]. In this view, cause occurs before effect and both are spatially contiguous. We use the model proposed by [14] to define and implement these constraints. According to this model, causal temporal relations can be classified into two main categories depending on the fact that $e_{c}$ occurs before or at the same time as $e_{e}$. The fact that the cause precedes the effect is due to threshold delays (for example, flooding will not occur before the water in the river increases beyond a certain level) or diffusion delays (cause and effect are not spatially co-located, and the cause takes some time to reach its effect). According to the causal relative spatial relation, cause must be spatially connected to its effect in either of two ways: undirected or directed connection. In addition, the connection's path must allow the propagation of a certain causing property, such as, for example, a lake does not allow the spread of fire [14].

Spatio-temporal constraints are necessary but not sufficient to infer that an event is a cause of another one. Other constraints must hold, referred to as causing property in [14], but they are not defined and represented in their model. We call these constraints semantic constraints. Semantic constraints refer to the qualitative causal knowledge that describes how entities composing the world influence each other. In our model, the world is composed of the geographic objects populating the $V G E$, the agents inserted in the $V G E$, and "Nature", represented by physical phenomena (Figure 6). Nature influences both the states of geographic objects (for example, rain makes the river flooded) and the states and - 
transitively- the behaviors of agents (for example, fog reduces the visibility field of a person, and then the person becomes more cautious when walking). The agents' behaviors may also depend on the states of geographic objects (for example, a person begins careful when crossing a wet floor). Finally, agents' behaviours lead to actions that may modify the states of geographic objects (for example, destroying a bridge). In our model we identify two types of semantic ontological causal knowledge. The first type describes the direct effects of changes introduced in the world, i.e. changes caused by agents' actions or by "Nature" (section 3.2.1.2). We use domain specific causal ontologies to represent knowledge about these two sources of change. An action executed by an agent has an effect that changes the state of the world, and so this action is viewed as the immediate cause of this change. Concerning changes that are caused by natural phenomena (for example, the characteristic of the fog in reducing visibility), their causal knowledge is embedded in the QS models (such as the Qualitative Process Theory [16]) that we use to simulate some physical phenomena in the geosimulation environment (section 3.2.1.2). We explicitly represent this knowledge in an ontology of physical natural phenomena. The second type of ontological causal knowledge describes how agents behave when some typical states of the world hold. With this causal knowledge we can for example infer that, in the event illustrated by Figure 5, thirst caused Hedi to enter the shop to look for a drink.

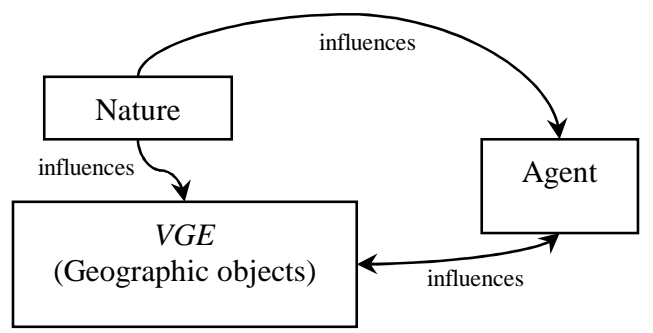

Figure 6. Entities of the world

\section{MAGS-COA}

MAGS-COA is a software that we developed - extending the MAGS environment [34] - as a proof of concept illustrating the approach that we propose to support a "What if" analysis of COAs (Figure 7).

In this system, the user starts by initializing the "What-if" experimentation. To do so, she first specifies the $V G E$ where the COA will be executed using the VGE Specification and Modification Module. This allows to initialize the GIS data describing the environment (different geo-objects and geoportions, their attributes and the relationships holding between them, defined in the Geographic Ontology) in the geosimulation module (Figure 7). She then uses the Agent Specification Module to select the resources participating in the experimentation and to locate them in the VGE. This allows the system to load the corresponding agents' models in the geosimulation module (Figure 7). Agents' models describe the attributes, the objectives and the elementary actions of the resources as defined in the Resources Ontology). See [34] for more details about agents' models in the MAGS platform. Geographic and Resources ontologies are specified in terms of conceptual graphs, using the Amine Platform [28]. After that, the user specifies the scenario describing the COA and the assumptions (using the Scenario specification Module). The COA specifies the sequence of tasks and the constraints imposed on the resources (the agents of the geosimulation) in order to achieve their mission. The assumptions are formalized as different "happenings" located in space and time (as for example, the beginning of wind blowing at a specific time and location).

Then, the user launches the geosimulation in the VGE. The resources of the COA are represented by autonomous software agents simulating the behaviors of the real resources. The happenings and their effects are simulated using qualitative simulation techniques, as mentioned in Section 3.2.1.2. The result of the geosimulation (simulation outcomes in Figure 7) is a set of events describing the sequence of changes occurring during the geosimulation and formalized as mentioned in Section 3.3.1.

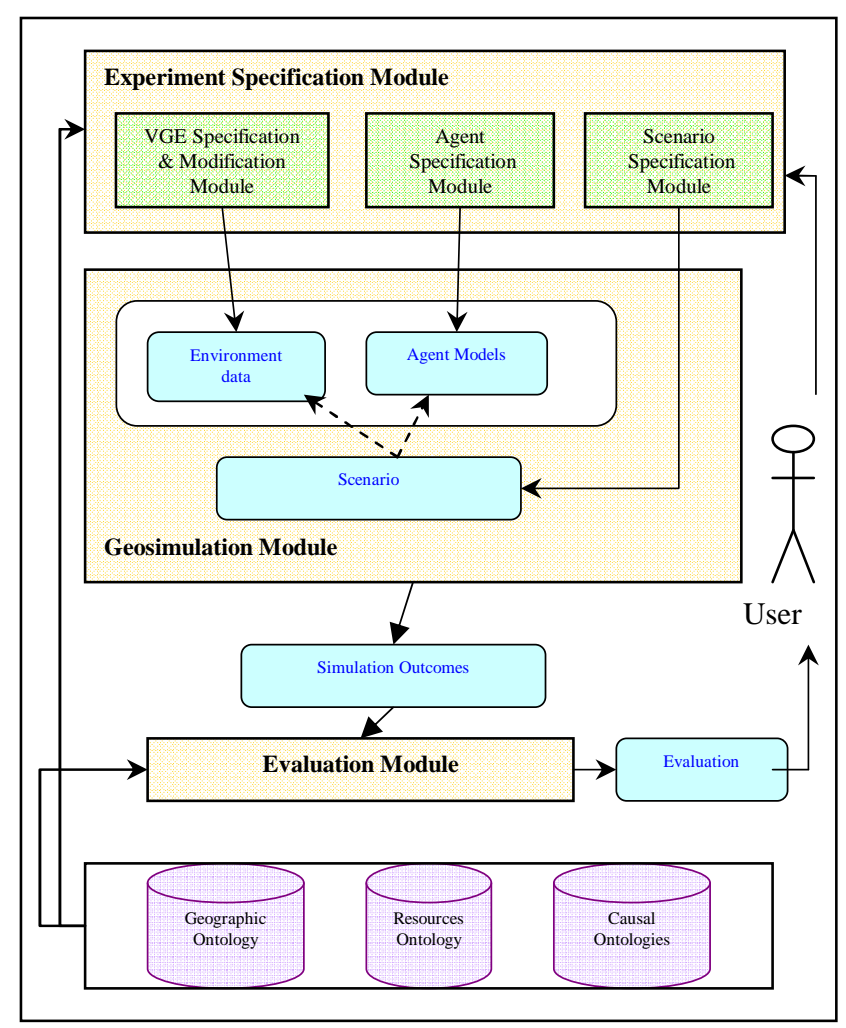

Figure 7. Architecture of MAGS-COA

The events resulting from the geosimulation are then analysed by the Evaluation module. The evaluation process consists of: 1) establishing a temporal ordering of the initial set of event instances (result of the geosimulation) and 2) for every pair of these events, verifying if the causal constraints hold. If these constraints hold, a new conceptual graph is created, making explicit the causal link between cause and effect events. If possible, a causal chain can be created between an initial cause and a final effect by including intermediate pairs of cause/effect events. The algorithms of this module are implemented using the Amine's Prolog+CG language which combines conceptual graphs, Prolog and Java programs. The results of the evaluation are recorded in a text file. 
Additional functionalities are proposed such as allowing the user to save an experiment in order to simulate it later and to add happenings during the simulation.

\section{APPLICATION DOMAIN, EXAMPLE AND RESULTS}

We applied our approach to a specific kind of decision support systems called critiquing systems. A critiquing system is a software that takes as an input a COA proposed by a human planner and gives as an output a qualitative assessment of the COA's weaknesses and strengths with respect to some criteria called critic dimensions [39]. A key feature of a critiquing system is its ability to give some explanations to show how it built its critics. This explanation capability distinguishes criticism from evaluation [20, 27], and consequently critiquing systems differ from pure evaluation tools. One way to apply critiquing systems to the "What if" analysis is to evaluate the plausibility of the user's assumptions.

We used MAGS-COA to implement a simple scenario and a realistic case study in the Search and Rescue $(S \& R)$ application domain, in which what-if analyses are effectively carried out. In this section we only illustrate the simple scenario with some details. The same techniques were used to develop the $S \& R$ scenario.

In the simple scenario, the COA specifies that three friendly CF18 planes must meet in a spatial zone $z l$, at a given time (Figure 8). After meeting there, they must create a formation (or a group) and go to zone $z 2$ to execute an attack operation on a precise target. During this operation, a leader plane is supposed to perform the attack while the two other planes must support it, i.e. protect it from possible attacks by enemy planes. In this kind of operation, time is a critical issue, and any delay may cause the failure of the operation. Let us test the following alternative: "What may happen if one of the friendly planes crosses an unexpected windy zone before reaching $z 1$ ?".

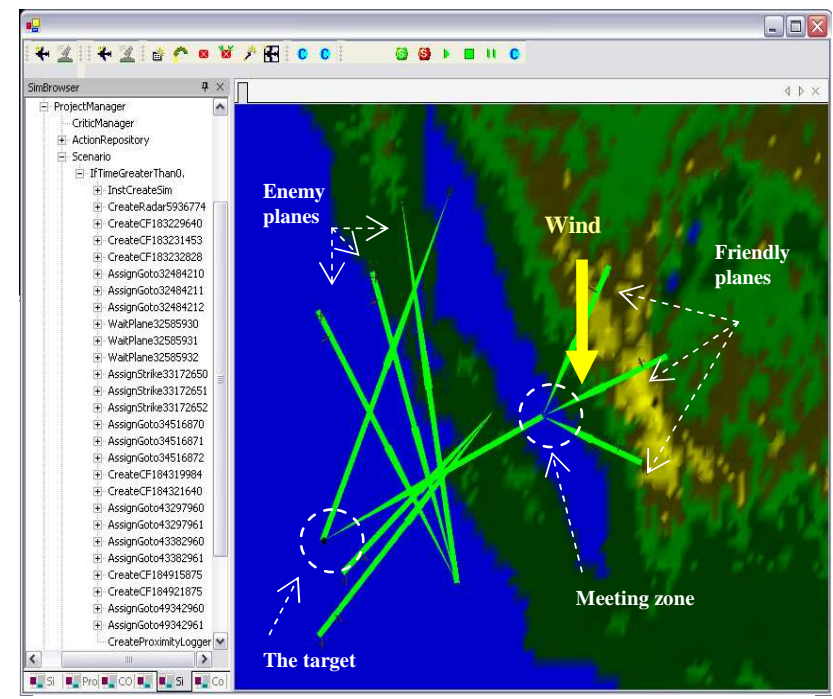

Figure 8. A scenario specification using MAGS-COA

We consider a qualitative definition of the wind, which is "a mass of air moving over the surface of the Earth in a definite direction" [1]. The wind "happening" is qualitatively simulated by a reactive object characterized by a spatial extent (dimensions and location), a speed and an orientation. A precise trajectory of the wind is not considered. As a first approximation, we use the "wind triangle" [36] to calculate the effect of the wind on planes. The wind triangle allows us to calculate the ground speed of the plane (the speed at which an aircraft is flying relative to the ground) in function of the airspeed (the speed at which an aircraft is flying relative to the air) and the windspeed. These three variables are modeled as vectors, and the ground speed vector is calculated by addition of airspeed and windspeed vectors. Using this model, we can calculate the effect of a Headwind (the wind movement is parallel to the aircraft's direction of motion but opposed to the aircraft's motion), a Tailwind (the wind movement is parallel to the aircraft's direction of motion and assists the aircraft's motion) or a Crosswind (the wind crosses the aircraft's path) on a plane. Thus, relative to the ground, the airplane would fly faster with a tailwind, slower with a headwind or drift right or left with a crosswind [1]. Figure 9 illustrates the example of a tailwind. Applying a vectors' addition, the tailwind will increase the ground speed of the plane. For example, if an airplane flies at $300 \mathrm{mph}$ with a tailwind of $40 \mathrm{mph}$, the ground speed of the plane is 340 mph $(300+40)$. Similarly, the wind vector allows to calculate the effect of a headwind and a crosswind on a plane. See $[1,35,36]$ for more details.

In the simulation, the effect of the wind is activated and calculated by the wind reactive agent (WRA). The WRA:

1) detects any new plane entering in its extent

2) compares its own orientation and the orientation of the plane in order to identify the rule to be applied (tailwind, headwind or crosswind)

3) modifies the variables ground speed and, in the case of a crosswind, orientation of the plane

These three steps represent the behaviour of the wind reactive agent in the simulation. The plane agent can carry out actions to compensate the wind effect, especially in the case of crosswinds. In this case, the plane agent- through its navigation algorithmuses other rules (examples can be found in [36]) to calculate and modify its new orientation.

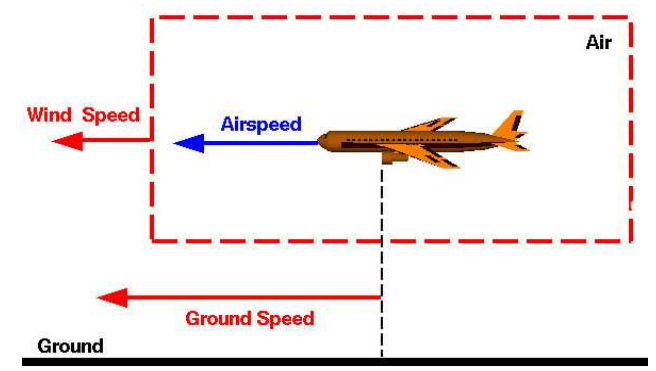

Figure 9. Effect of a tailwind: Ground speed = Vector sum of Airspeed and Windspeed = Airspeed + Windspeed (photo taken from [35])

Figure 10 illustrates an example of an event produced by the observer agents during the geosimulation (simulation outcomes). The example describes the event representing the fact that the plane CF18-03 enters zone14 and that, at the same time, its orientation changes. As stated in Section 3.3.2, the event does not 
explain why the plane orientation changes. This must be inferred by the evaluation module.

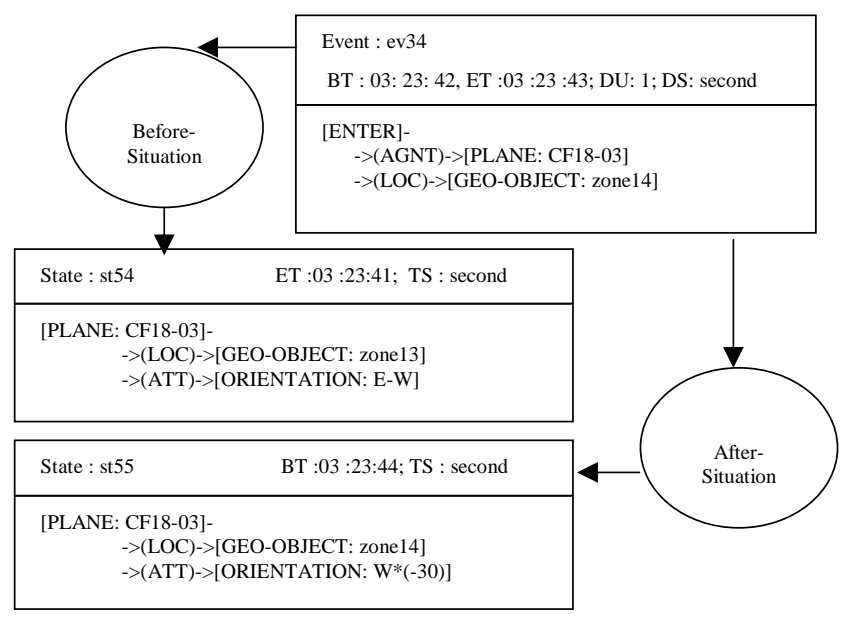

Figure 10. An event of the simulation formalized in CG

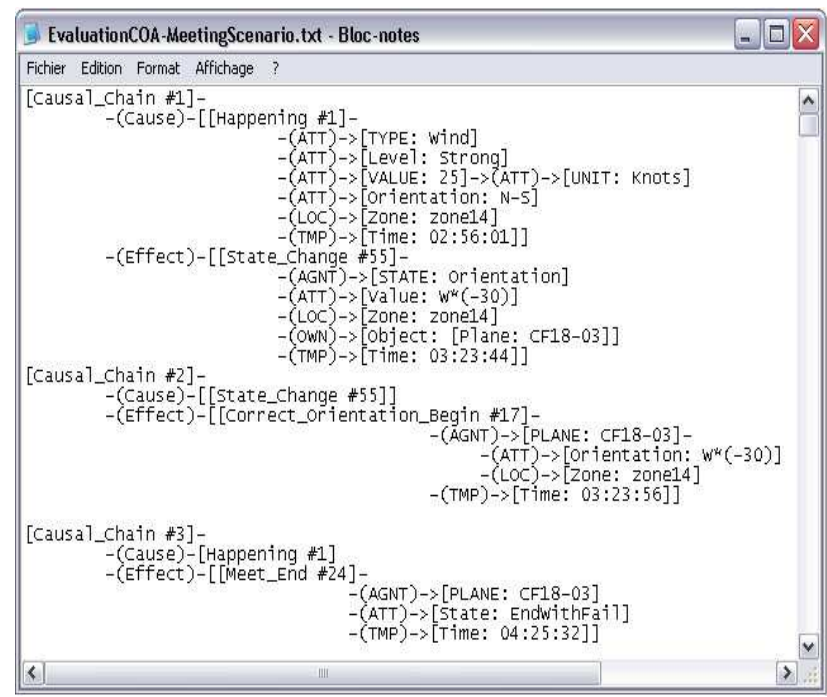

Figure 11. An evaluation of the scenario

Figure 11 illustrates the text file produced by the evaluation module for this scenario. The CG "[Causal_Chain \#1]" describes that the strong wind (with orientation $\mathrm{N}-\mathrm{S}$ ) occurring in the zone zone14 ([Happening \#1]) modified the orientation of the plane CF18-03 ("[State_Change \#55]"). This conclusion is inferred using causal constraints as mentioned in Section 3.3.2. In fact, the event Happening \#1 occurred before the event State_Change \#55, and both of them occurred in the location zone1 $\overline{4}$, so the two events satisfy the spatio-temproal constraints of causal relationships. In addition, in the causal knowledge it is specified that wind can either modify the orientation of flying objects or modify their orientation, and that planes are flying objects. This leads to the fact that causal semantic constraints hold between the two events. A similar reasoning processes leads the system to infer, in "[Causal_Chain \#2]", that the plane tried to maintain its initial orientation by executing the "Correct_Orientation" action
(“[Correct_Orientation_Begin \#17]”), but it failed to reach zone $z 2$ at the specified time ("[Meet_End \#24]"). In "[Causal_Chain \#3]" the evaluation shows that the wind was the cause of this delay (using some other causal knowledge, such as "windy zones may be an obstacle for flying objects" and "avoiding an obstacle may cause delay").

Compared to existing COAs critiquing systems, our results are promising. First, we use realistic GIS data to structure our VGE. Current COA critiquing systems often reason about non-realistic qualitative representations of space such as sketches [18], coarse drawn maps presenting spatial regions and the location of assets in these regions $[8,11]$. Second, our approach allows performing a causal analysis of a dynamic representation of a COA. Current COA evaluation systems reason about a static representation of space, and causal analysis is not performed. The only dynamic aspect represented by some of these systems is related to the mobility of COA assets [4]. Moreover, and as a consequence of this limit, current COA evaluation systems need more effort to be able to "produce non-obvious critics that add value to what a user can quickly determine with a visual inspection of a COA sketch" [4]. Indeed, it is known that human mental representation of space presents several limits, such as difficulties to judge distances, to identify directions, and to estimate the three-dimensional aspects of the geographic space [44]. By using agent-based geosimulation, our approach is expected to be helpful by alleviating the mental simulation of the human decision maker and remedying to his limits of mental spatial representation.

\section{DISCUSSION AND FUTURE WORKS}

In this paper we proposed a multiagent geosimulation-based approach to support the "What if" analysis of COAs in a geographic context. An innovative part of our approach is the new qualitative technique that we propose for data analysis. In fact, simulation results are usually analyzed using statistical and mathematical models. The main problem with these techniques is that only users with mathematical or statistical backgrounds can understand them and derive interesting conclusions. In addition, these techniques are only appropriate to analyze recurrent phenomena. In the context of a qualitative evaluation - such as a "what if" analysis - we need to express results using the concepts and language understood by users, especially by novice ones. In addition, we need to only highlight the pertinent elements and to take the user away from non relevant details. We think that our approach meets these requirements. In fact, by using ontologies, we can express results using the concepts understood by the user. In addition, the use of observer agents allows the system to collect data about the sole phenomena that are relevant to the user. Moreover, this data is extracted from a somewhat realistic and precise representation of the geographic realm which is embedded in the multiagent geosimulation environment which is a natural way for a user to inspect a realistic situation. Finally, there is a mapping between CGs and natural language; CGs are considered as an intermediate language for translating computer-oriented formalisms to and from natural languages [24]. By formalizing the simulation results using CGs, it is possible to express them using natural language.

Another innovative part of our approach is to explicitly model and simulate dynamic phenomena in geographic environments combining multiagent geosimulation and Qualitative Simulation (QS). In fact, we may distinguish two kinds of spatial dynamic 
phenomena in the literature. On the one hand, there are phenomena representing only behaviours of agents in spatial context. Mobility is one of the most analyzed behaviour, especially in the transportation domain. These phenomena are usually modeled and analysed using multiagent geosimulation approaches, such as in $[19,26]$. In these approaches, dynamic phenomena are not modeled as entities in their own right. On the other hand, there are spatial dynamic phenomena involving only physical natural processes. These phenomena have been widely studied by QS research communities, such as the Qualitative Process Theory [16] and the Potential Field Theory based QS [32]. The models proposed by the QS research community cannot take into account dynamic situations involving autonomous agents. By combining both multiagent geosimulation and QS techniques, our approach allows to explicitly model, simulate and analyze dynamic geographical phenomena involving natural activities and agents.

Our approach was judged promising by experts, and we are currently working on validating the results of the $S \& R$ case study with them and we try to determine if the approach really has an added value to them. In the next months, we plan to try our approach on other scenarios involving a larger number of agents and happenings.

\section{ACKNOWLEDGMENTS}

This research is supported by GEOIDE, the Canadian Network of Centers of Excellence in Geomatics (MUSCAMAGS Project), by the Canadian National Sciences and Engineering Research Council, and by Valcartier Defence R\&D Canada (DRDC), Quebec, Canada (MAGS-COA Project).

\section{REFERENCES}

[1] Aero Training Products. NAVIGATION, chapter 8 of Pilot's Handbook of Aeronautical Knowledge (Canadian Edition, an ATP's FREE On-line Edition, http://www.aerotraining.com/reference/AC\%206123C_Chapter_8_Canada.pdf

[2] Ali, W. and Moulin, B. 2005. 2D-3D MultiAgent GeoSimulation with Knowledge-Based Agents of Customers' Shopping Behavior in a Shopping Mall, LNCS 3693, Springer Berlin Heidelberg (2005), 445-458

[3] Arizona National Guard web site, 2005 presentation, http://www.azguard.gov/AZAASF1/Trainingfiles/azaasf1aca d\&trng.htm

[4] Barker, K., Blythe, J., Borchardt, G., Chaudhri, V.K., Clark, P.E., Cohen, P., Fitzgerald, J., Forbus, K., Gil, Y., Katz, B., Kim, J., King, G., Mishra, S., Morrison, C., Murray, K., Otstott, C., Porter, B., Schrag, R.C., Uribe, T., Usher, J. and Yeh, P.Z. 2003. A Knowledge Acquisition Tool for Course of Action Analysis. In Proceedings of the innovative Applications of Artificial Intelligence Conference, (2003)

[5] Batty, M., Desyllas, J. and Duxbury, E. 2003. The discrete dynamics of small-scale spatial events: agent-based models of mobility in carnivals and street parades. International Journal of Geographical Information Science (2003), 17(7), 673-697

[6] Benenson, I. and Torrens, P.M. (Eds.) 2004. Geosimulation: Object-Based Modelling of Urban Phenomena. Special
Issue: Computers, Environment and Urban Systems, 28 (1/2) (2004)

[7] Bhatt, M. 2007. A causal approach for modeling spatial dynamics: a preliminary report. Workshop on Spatial and Temporal Reasoning, IJCAI (2007)

[8] Boicu, M., Tecuci, G., Marcu, D., Stanescu, B., Boicu, C., Balan, C., Barbulescu, M. and Hao, X. 2002. DiscipleRKF/COG: Agent Teaching by Subject Matter Experts. In the Fourteenth Conference on Innovative Applications of Artificial Intelligence, Edmonton, Alberta, Canada, AAAI Press/The MIT Press 2002, 992 - 993

[9] Bowman, M., Lopez, A., and Tecuci, G. 2001. Ontology Development for Military Applications. Proceedings of the Thirty-ninth Annual ACM Southeast (2001)

[10] Brown, J.R. 2002. Thought experiments. The Stanford Encyclopedia of Philosophy, (2002)

[11] Chandrasekaran, B., Josephson, J.R., Banerjee, B. and Kurup, U. 2002. Diagrammatic Reasoning in Support of Situation Understanding and Planning. Proceedings of Army Science Conference, Dec 2-5, Orlando, Fl (2002)

[12] Cohn, A., and Hazarika, S. 2001. Qualitative Spatial Representation and Reasoning: An Overview. Fundamental Informaticae, Vol. 46 (1-2), (2001), 1-29

[13] Desclés, J.-P. 1990. Langages applicatifs, langues naturelles et cognition. Hermès, Paris (1990)

[14] El-Geresy, B., Abdelmoty, A. and Jones, C. 2002. SpatioTemproal Geographic Infromation Systems: A causal Perspective. In Y. Manolopoulos and P. Navrat (Eds.), ADBIS 2002, LNCA 2435, Springer-Verlag (2002), 191-203

[15] Fonseca, F., Egenhofer, M., Davis, C. and Câmara, G. 2002. Semantic Granularity in Ontology-Driven Geographic Information System. Annals of Mathematics and Artificial Intelligence, Vol. 36 (1-2) (2002), 121-151

[16] Forbus, K.D. 1984. Qualitative Process Theory. Artificial Intelligence (1984), (24), 85-168

[17] Forbus, K.D. 2002. Qualitative modeling of common sense understanding. Cognitive Science Society Virtual Colloquium Series, (2002)

[18] Forbus, K.D., Usher, J. and Chapman, V. 2003. Sketching for military Courses of Action Diagrams. Proceedings of IUI'03, Miami, Florida (2003)

[19] Frihida, A., Marceau, D. J. and Theriault, M. 2004. Extracting and Visualizing Individual Space-Time Paths: An integration of GIS and KDD in Transport Demand Modelling. Cartography and Geographic Information Science, 31(1), 19-28 (2004)

[20] Govier T. 2000. Critical review: Manifest Rationality; A Pragmatic Theory of Argument. Informal logic (2000), 20, 281-291

[21] Grenon, P. and Smith, B. 2004. SNAP and SPAN: Towards dynamic spatial ontology. Spatial Cognition and Computation, Vol. 4 (1) (2004), 69-103

[22] Haddad, H. and Moulin, B. 2007. Using Cognitive Archetypes and Conceptual Graphs to Model Dynamic Phenomena in Spatial Environments. In U. Priss, S. 
Polovina, and R. Hill (Eds.): ICCS 2007, LNAI 4604, Springer-Verlag Berlin Heidelberg (2007), 69-82

[23] Haddad, H. and Moulin, B. 2008. A Framework to Model, Simulate and Qualitatively Analyse Courses of Actions in Dynamic Virtual Geographical Environments. Accepted for publication with revisions in Spatial Cognition and Computation, November 24, 2007

[24] Hensman, S. and Dunnion, J. 2004. Using Linguistic Resources to Construct Conceptual Graph Representation of Texts. Text chapter Text, Speech and Dialogue , vol. 3206 (2004), 81-88

[25] Herath, S. 2001. Geographical Information Systems in Disaster Reduction. Information Technology For Disaster Management, No.1 (2001), 25-31

[26] Isenegger, D., Price, B., Wu, Y., Fischlin, A., Frei, U. and Weibel, R. 2005. IPODLAS--A software architecture for coupling temporal simulation systems, VR, and GIS. ISPRS Journal of Photogrammetry and Remote Sensing, 60(1) (2005), 34-47

[27] Johnson, R.H and Blair, A.J. 2000. Informal logic: An Overview. Informal logic, 20(2) (2000), 93-107

[28] Kabbaj, A., Bouzouba, K., El Hachimi, K. and Ourdani, N. 2006. Ontologies in Amine Platform: Structures and Processes. Proceedings of ICCS (2006), LNAI Vol. 4068, 300-313

[29] Kitamura, Y., Ikeda, M. and Mizoguchi, R. 1997. A Causal Time Ontology for Qualitative Reasoning. In the Fifteenth International Joint Conference on Artificial Intelligence (1997) IJCAI-97, 501-506

[30] Koch, A. 2000. Linking Multi-Agent Systems and GIS Modeling and simulating spatial interactions, Department of Geography RWTH Aachen. Angewandte Geographische Informationsverarbeitung XII, Beiträge zum AGITSymposium Salzburg 2000, Hrsg.: Strobl/Blaschke/Griesebner, Heidelberg, p. 252-262

[31] Kuipers, B. 2001. Qualitative Simulation. In Encyclopedia of Physical Science and Technology, Third Edition. Robert A. Meyers, Editor-in-Chief. Academic Press (2001), 287-300

[32] Lundell, M. 1996. A Qualitative Model of Physical Fields, National Conference on Artificial Intelligence (AAAI), Portland, USA, 1996

[33] Mark, D., Smith, B. and Tversky, B. 1999. Ontology and geographic objects: An empirical study of cognitive categorization. In C Freksa and David Mark, editors, Spatial Information Theory. Cognitive and Computational Foundations of Geographic Information Science (1999), LNCS 1661, 283-298

[34] Moulin, B., Chaker, W., Perron, J., Pelletier, P., Hogan, J. and Gbei, E. 2003. MAGS Project: Multi-agent geosimulation and crowd simulation. In the proceedings of the COSIT'03 Conference: Spatial Information Theory. LNCS, Vol. 2825, 151-168, Springer Verlag (2003)

[35] NASA 2006. Guided Tours to the Beginner's Guide to Aeronautics, http://www.grc.nasa.gov/WWW/K12/airplane/move.html, Lust update: Oct 052006

[36] Recreational Aviation Australia Inc. 2007. Flight Planning and Navigation Guide, last changed May 30, 2007 http://www.auf.asn.au/navigation/index.html

[37] Shen, D.Y., Takara, K., Tachikawa, Y. and Liu, Y.L. 2006. 3D simulation of soft geo-objects. International Journal of Geographical Information Science (2006), Vol. 20, No. 3, 261-271

[38] Shoham, Y. and Goyal, N. 1988: Temporal reasoning in artificial intelligence. In H.E. Shorbe (Ed.), Exploring Artificial Intelligence: Survey Talks from the National Conferences on Artificial Intelligence (1988), 419-438

[39] Silverman, B.G. 1992. Survey of Expert Critiquing Systems: Practical and Theoretical Frontiers. Communication of the ACM (1992), Vol.35, No.4

[40] Sowa, J.F. 1984. Conceptual Structures: Information Processing in Mind and Machine. Adison-Wesley, Massachusetts (1984)

[41] Torrens, P.M. 2006. Geosimulation and its application to urban growth modeling. In Complex Artificial Environments, J. Portugali (Eds), London: Springer-Verlag (2006), 119-134

[42] Torrens, P.M. 2007. Behavioral intelligence for geospatial agents in urban environments, IEEE Intelligent Agent Technology (IAT 2007). Los Alamitos, CA, IEEE, pp. 63-66

[43] Trickett, S. and Trafton, J. G. 2007. "What if": The Use of Conceptual Simulations in Scientific Reasoning. Cognitive Science (2007), (31) 843-875

[44] Tversky, B. 2005. Visuospatial reasoning. Chapter 10 in K. Holyoak and R. Morrison (Eds.), The Cambridge Handbook of Thinking and Reasoning. Cambridge, MA: Cambridge University Press. (2005)

[45] Westervelt, J. D., and Hopkins, L. D. 1999. Modeling mobile individuals in dynamic landscapes. International Journal of Geographical Information Science (1999), 13(3), 191-208

[46] Wolff, P. 2007. Representing Causation. In [2007] Workshop: Causality, Mechanisms, and Psychology, Pittsburgh, PA; (2007)

[47] Worboys, M. and Hornsby, K. 2004. From objects to events: GEM, the geospatial event model. 3d Internatinal Conference on GIScience. Vol. 3234. LNCS, 327-344, Springer. Berlin (2004) 